The Astrophysical Journal, in Press

Preprint typeset using $\mathrm{LATE}_{\mathrm{E}} \mathrm{X}$ style emulateapj v. 08/13/06

\title{
SPECTROPOLARIMETRY OF EXTREMELY LUMINOUS TYPE Ia SUPERNOVA 2009dc: NEARLY SPHERICAL EXPLOSION OF SUPER-CHANDRASEKHAR MASS WHITE DWARF ${ }^{1}$
}

\author{
Masaomi Tanaka $^{2,3}$, Koji S. Kawabata ${ }^{4}$, Masayuki Yamanaka ${ }^{5,4}$, Keitchi Maeda ${ }^{3}$, Takashi Hattori ${ }^{6}$, Kentaro \\ Aoki $^{6}$, Ken'ichi Nomoto ${ }^{3}$, Masanori Iye ${ }^{7}$, Toshiyuki Sasaki ${ }^{6}$, Paolo A. Mazzali ${ }^{8,9}$, and Elena Pian ${ }^{10,11}$ \\ The Astrophysical Journal, in press
}

\begin{abstract}
We present the first spectropolarimetric observations of a candidate of super-Chandrasekhar mass Type Ia supernova (SN): SN 2009dc. The observations were performed at 5.6 and 89.5 days after the $B$-band maximum. The data taken at the later epoch are used to determine the interstellar polarization. Continuum polarization is found to be small $(<0.3 \%)$, indicating that the explosion is nearly spherically symmetric. This fact suggests that a very aspherical explosion is not a likely scenario for SN 2009dc. Polarization at the Si II and Ca II lines clearly shows a loop in the $Q-U$ plane, indicating a non-axisymmetric, clumpy distribution of intermediate-mass elements. The degree of line polarization at the $\mathrm{Si}$ and $\mathrm{Ca}$ line is moderate $(0.5 \% \pm 0.1 \%$ and $0.7 \% \pm 0.1 \%$, respectively), but it is higher than expected from the trend of other Type Ia SNe. This may suggest that there are thick enough, clumpy Si-rich layers above the thick ${ }^{56} \mathrm{Ni}$-rich layers $\left(\gtrsim 1.2 M_{\odot}\right)$. The observed spectropolarimetric properties, combined with the photometric and spectroscopic properties, suggest that the progenitor of SN $2009 \mathrm{dc}$ has a super-Chandrasekhar mass, and that the explosion geometry is globally spherically symmetric, with clumpy distribution of intermediate-mass elements.

Subject headings: polarization — supernovae: general — supernovae: individual (SN 2009dc) supernovae: individual (SNe 2003fg, 2006gz, 1991T) — white dwarfs
\end{abstract}

\section{INTRODUCTION}

Type Ia supernova (SN) is a thermonuclear explosion of a $\mathrm{C}+\mathrm{O}$ white dwarf in a close binary (see, Hillebrandt \& Niemever 2000; Nomoto et al. 1994, for a review). When the mass of the white dwarf becomes close to the Chandrasekhar mass $\left(\simeq 1.38 M_{\odot}\right)$, the explosion is triggered. Thus, the ejecta mass of Type Ia $\mathrm{SNe}$ is always similar (Mazzali et al. 2007). Accordingly, the observed properties of Type Ia SNe are more homogeneous than those of core-collapse $\mathrm{SNe}$, which have various ejecta masses.

Thanks to this homogeneity, Type Ia SNe are used as a cosmological distance indicator. Although it is known that the maximum luminosity has a diversity, this can be corrected using the shape of the light curve: the brighter SNe decline more slowly (e.g., Phillips 1993;

\footnotetext{
${ }^{1}$ Based on data collected at Subaru Telescope, which is operated by the National Astronomical Observatory of Japan.

2 Department of Astronomy, Graduate School of Science, University of Tokyo, Bunkyo-ku, Tokyo, Japan; mtanaka@astron.s.utokyo.ac.jp

${ }^{3}$ Institute for the Physics and Mathematics of the Universe, University of Tokyo, Kashiwa, Japan

${ }^{4}$ Hiroshima Astrophysical Science Center, Hiroshima University, Higashi-Hiroshima, Hiroshima, Japan

${ }_{5}$ Department of Physical Science, Hiroshima University, Higashi-Hiroshima, Hiroshima, Japan

${ }^{6}$ Subaru Telescope, National Astronomical Observatory of Japan, Hilo, HI

7 Optical and Infrared Astronomy Division, National Astronomical Observatory, Mitaka, Tokyo, Japan

8 Max-Planck Institut für Astrophysik, Karl-SchwarzschildStrasse 2 D-85748 Garching bei München, Germany

${ }^{9}$ Istituto Naz. di Astrofisica-Oss. Astron., vicolo dell'Osservatorio, 5, 35122 Padova, Italy

10 Istituto Naz. di Astrofisica-Oss. Astron., Via Tiepolo, 11, 34131 Trieste, Italy

${ }^{11}$ European Southern Observatory, Karl-Schwarzschild-Strasse 2, D-85748, Garching, Germany
}

Phillips et al. 1999). Using Type Ia SNe as a distance indicator, the accelerating nature of the Universe has been revealed (e.g., Riess et al. 1998; Perlmutter et al. 1999).

Recently, the discovery of a class of extremely luminous Type Ia SNe has casted the question on the progenitor scenario. Howell et al. (2006) reported that SN $2003 \mathrm{fg}$ had a maximum brightness $M_{V}=-19.9$ mag. To power such an extreme luminosity, $\sim 1.3 M_{\odot}$ of ${ }^{56} \mathrm{Ni}$ is required. Despite the large ${ }^{56} \mathrm{Ni}$ production, the expansion velocity of SN $2003 \mathrm{fg}\left(\sim 8,000 \mathrm{~km} \mathrm{~s}^{-1}\right.$ near the maximum brightness) is slower than normal Type Ia SNe $(\sim 10,000$ $\mathrm{km} \mathrm{s}^{-1}$ ). From these facts, they suggested that the progenitor of SN 2003fg has a super-Chandrasekhar mass $\left(\sim 2 M_{\odot}\right)$.

Another candidate of super-Chandrasekhar mass Type Ia SN is SN 2006gz (Hicken et al. 2007). The maximum brightness is $M_{V} \sim-19.74$ mag when the host extinction $A_{V}=0.56 \mathrm{mag}$ is assumed. SN 2007if also shows spectral similarities to SN 2003fg (see Yuan et al. 2007; Akerlof et al. 2007; Rau et al. 2007; Scalzo et al. 2010).

This class of objects commonly shows clear C II lines in the optical spectrum. The $\mathrm{C}$ lines are rarely seen in normal Type Ia SNe (Marion et al. 2006; Tanaka et al. 2008b). The presence of $\mathrm{C}$ also seems to support a super-Chandrasekhar mass progenitor since it suggests that there is a lot of unburned elements above the thick ${ }^{56} \mathrm{Ni}$-rich layer. The C-rich ejecta might also explain the faintness of SN 2006gz at late phases (Maeda et al. 2009).

However, there is a possibility that this class of objects is an aspherical explosion of a Chandrasekhar mass white dwarf. Hillebrandt et al. (2007) discussed the difficulty in synthesizing much ${ }^{56} \mathrm{Ni}$ in a differentially rotating white dwarf, and suggested an aspherical, lopsided explosion of a Chandrasekhar mass white dwarf. Maeda \& Iwamoto (2009) argued that a combination of the low velocity and the moderate light curve de- 
cline rate of SN $2003 \mathrm{fg}$ is not compatible with superChandrasekhar mass explosion, although the observed properties of SN 2006gz are consistent with a spherical explosion of a super-Chandrasekhar mass progenitor. They also suggested possible asphericity, if those two overluminous SNe are to be explained in a unified scenario. Asphericity must be one of the keys to understanding the nature of the extremely luminous Type Ia SNe.

Asphericity of extragalactic SNe can be studied by polarization measurements (see Wang \& Wheeler 2008, for a review). Polarization is generated by the electron scattering in the SN ejecta. When a photon is scattered by an electron with nearly $90^{\circ}$, unpolarized light gains large linear polarization, whose vector direction is orthogonal to the scattering plane. Since extragalactic SNe are point sources, the polarization vectors are cancelled out in spherically symmetric ejecta. Thus, if polarization is detected, it undoubtedly means asymmetry of the SN ejecta (Shapiro \& Sutherland 1982; McCall 1984; Höflich 1991). The larger degree of polarization means larger deviation from spherical symmetry. Past observations show that the continuum polarization of normal Type Ia SNe is small, being $\lesssim 0.3 \%$ (Wang et al. 1997, 2003a, 2006; Leonard et al. 2005; Chornock \& Filippenko 2008). Thus, the explosion of normal Type Ia SNe is almost spherical.

In addition, polarization at the line has information on element distribution. Scattering by lines tends to depolarize lights (Jeffrev 1989; Kasen et al. 2003). If the line scattering elements are distributed aspherically, the cancellation of polarization vectors becomes incomplete at the absorption minimum of the P Cygni profile. As a result, enhancement of the polarization degree is detected at the absorption. In other words, detection of the line polarization directly means that the distribution of the elements is not uniform in front of the photosphere.

Although spectropolarimetry is a powerful tool, it was not performed for the three candidates of superChandrasekhar mass Type Ia SN so far. In this paper, we present the first spectropolarimetric observation of a candidate of super-Chandrasekhar mass Type Ia SN: SN $2009 \mathrm{dc}$

SN 2009dc was discovered by Puckett et al. (2009) in S0 galaxy UGC 10064. Harutyunyan et al. (2009) performed the first spectroscopy and reported the slow expansion velocity and the presence of the $\mathrm{C}$ II line. Marion et al. (2009) performed infrared spectroscopy and reported non-detection of $\mathrm{C}$ I lines.

Yamanaka et al. (2009) showed that SN 2009dc is an extremely luminous event. If the distance modulus $\mu=34.88 \mathrm{mag}$ and Galactic extinction $A_{V}=0.22 \mathrm{mag}$ (Schlegel et al. 1998) are assumed, the peak absolute magnitude is $M_{V}=-19.9 \mathrm{mag}$ (even assuming null extinction in the host galaxy). This is twice as bright as the average luminosity of Type Ia SNe. The required mass of ${ }^{56} \mathrm{Ni}$ is estimated to be $\gtrsim 1.2 M_{\odot}$. Thus, SN $2009 \mathrm{dc}$ belongs to the same class as SNe 2003fg and 2006gz: the fourth candidate of super-Chandrasekhar mass Type Ia SN. For the properties of SN $2009 \mathrm{dc}$, see also a recent paper by Silverman et al. (2010).

In this paper, we report the spectropolarimetric observations of SN 2009dc and study the explosion geometry and the progenitor of this SN. In Section 2 , we describe our observation and data reduction. In Section 3, we show the results of the spectropolarimetry. An estimate for interstellar polarization is also given. We summarize the intrinsic properties of continuum and line polarization of SN 2009dc in Section 4 Our spectropolarimetric observations suggest that SN 2009dc is a nearly spherical explosion of a super-Chandrasekhar mass white dwarf.

\section{OBSERVATIONS AND DATA REDUCTION}

Spectropolarimetric observations of SN 2009dc were performed with the $8.2 \mathrm{~m}$ Subaru telescope equipped with Faint Object Camera and Spectrograph (FOCAS, Kashikawa et al. 2002) on UT 2009 May 1.5 $(\mathrm{MJD}=54952.5)$ and July 24.4 (MJD=55036.4). These epochs correspond to $t=+5.6$ and +89.5 days from the $B$ band maximum (MJD=54946.9, Yamanaka et al. 2009). Hereafter, $t$ denotes the days after the $B$-band maximum in the observer's frame.

For the observation at $t=+5.6$ days, we used an offset slit of $0.8^{\prime \prime}$ width, a 300 lines $\mathrm{mm}^{-1}$ grism, and the Y47 filter. This configuration gives a rather wide wavelength coverage, 4400-9000 $\AA$. At $t=+89.5$ days, given the expected small polarization level, we used a center slit of $0.8^{\prime \prime}$ width (with the same grism without any ordersorting filter) since the instrumental polarization of FOCAS is negligible $(\lesssim 0.1 \%)$ when the center slit is used (Tanaka et al. 2008a). This configuration gives a wavelength coverage of 4000-7000 A. The wavelength resolution is $\lambda / \Delta \lambda \sim 650$ in both settings.

For the measurement of linear polarization, FOCAS is equipped with a rotating superachromatic half-wave plate and a crystal quartz Wollaston prism. An incident beam is split to ordinary and extraordinary lights by the Wollaston prism, and both rays are recorded on the CCD simultaneously. One set of observations consists of the integration with $0^{\circ}, 45 .^{\circ}, 22.5^{\circ}$, and $67.5^{\circ}$ positions of the half-wave plate. From this one set of exposures, Stokes parameters $Q$ and $U$ can be derived as described by Tinbergen (1996).

For the observations at $t=+5.6$ days, we performed three sets of four integrations with the exposure time of $2400 \mathrm{~s}$ for each set $(600 \mathrm{~s} \times 4)$. Also, one set was taken with the exposure time of $3600 \mathrm{~s}(900 \mathrm{~s} \times 4)$. At $t=+89.5$ days, we took three sets of four integrations with the exposure time of $3200 \mathrm{~s}$ for each set $(800 \mathrm{~s} \times$ 4). The total exposure time is $10800 \mathrm{~s}$ and $9600 \mathrm{~s}$ at $t=+5.6$ and +89.5 days, respectively.

We derived Stokes parameters for each set of observations independently. And then, these were combined, rejecting the apparent noises (by rejecting $>3 \sigma$ points from the median of 30-50 $\AA$ window, and by further visual inspection). When the offset slit is used $(t=+5.6$ days), the instrumental polarization of $\sim 0-0.4 \%$ is corrected by observing the unpolarized stars $\mathrm{BD}+32^{\circ} 3739$ and $\mathrm{BD}+28^{\circ} 4211$ (Schmidt et al. 1992). The reference axis of the position angle was calibrated by the observation of the strongly polarized star HD 155197 (Turnshek et al. 1990). The wavelength dependence of the optical axis of the half-wave plate was corrected using the dome flat taken through a fully-polarizing filter.

The total flux was calibrated using the observation of the spectrophotometric standard star BD $+28^{\circ} 4211$ Oke 


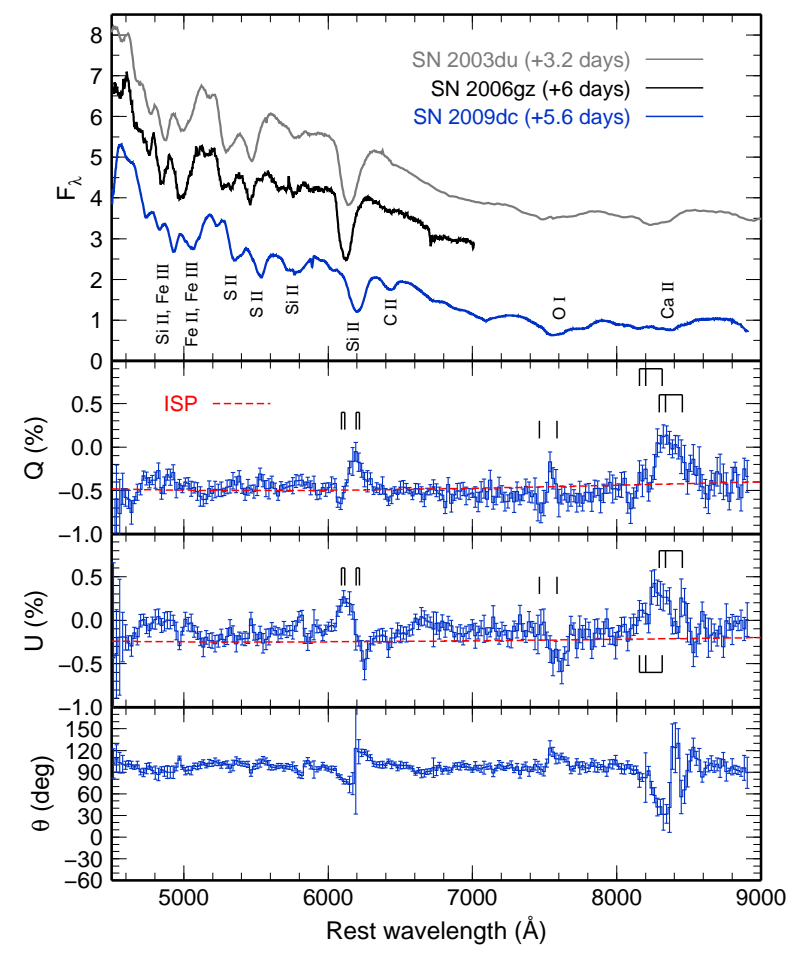

FIG. 1. - Total flux and polarization spectrum of SN 2009dc at $t=+5.6$ days (blue lines). In polarization spectrum, ISP is not corrected for. Polarization data are binned into $20 \AA$. In the top panel, the total flux of SN $2009 \mathrm{dc}$ (in $10^{-15} \mathrm{erg} \mathrm{s}^{-1} \mathrm{~cm}^{-2} \AA^{-1}$ ) is compared with the normal Type Ia SN 2003du (scaled flux, shifted by 3.0, Stanishev et al. 2007) and the overluminous Type Ia SN 2006gz (scaled flux, shifted by 1.5, [Hicken et al. [2007). The red dashed lines show the estimated ISP (Section 3.3). Vertical lines at the Si II $(\lambda 6347,6371)$, O I $\lambda 7774$, and Ca II $(\lambda 8498,8542,8662)$ lines show the $7,200 \mathrm{~km} \mathrm{~s}^{-1}$ and $12,000 \mathrm{~km} \mathrm{~s}^{-1}$ positions.

1990) at both epochs. Telluric absorption lines were also removed using the spectrum of this standard star.

Throughout this paper, we define Stokes parameters as a fraction of the total flux: $Q \equiv \hat{Q} / I$ and $U \equiv \hat{U} / I$, where $\hat{Q}$ and $\hat{U}$ can be expressed by $\hat{Q}=I_{0}-I_{90}$ and $\hat{U}=I_{45}-I_{135}$, respectively. Here $I_{\theta}$ is the intensity measured through the ideal polarization filter with an angle $\theta$ (see e.g., Landi degl'Innocenti 2002). The angle is measured from north to east. From Stokes $Q$ and $U$, the position angle is obtained by $\theta \equiv 0.5 \operatorname{atan}(U / Q)$. The degree of total polarization is $P \equiv \sqrt{Q^{2}+U^{2}}$. Because of this definition, total polarization is biased in a positive direction. This bias is corrected using the results of Patat \& Romaniello (2006) when $P$ is shown.

\section{RESULTS}

\subsection{Spectroscopic Properties}

The top panel of Figure 1 shows the total flux spectrum of SN 2009dc at $t=+5.6$ days (blue) compared with the spectra of normal SN 2003du (gray, Stanishev et al. 2007) and overluminous SN 2006gz (black, Hicken et al. 2007). SN 2003du is a well-studied normal Type Ia SN $(z=0.00638$, Gerardy et al. 2004; Anupama et al. 2005; Stanishev et al. 2007). SN 2006gz is an overluminous Type Ia SN, which is a candidate for superChandrasekhar mass SN $(z=0.0236$, Hicken et al.



FIG. 2.- Total flux and polarization spectrum of SN 2009dc at $t=+89.5$ days (blue lines). In polarization spectrum, ISP is not corrected for. Polarization data are binned into $100 \AA$. In the top panel, the total flux of SN 2009dc (in $10^{-16} \mathrm{erg} \mathrm{s}^{-1} \mathrm{~cm}^{-2} \AA^{-1}$ ) is compared with the normal Type Ia SN 2003du (scaled flux, shifted by 2.0, Stanishev et al. 2007). The red dashed lines show the estimated ISP (Section 3.3).

2007). Compared with normal Type Ia SNe, the spectrum of SN 2009dc is unique in the following points: (1) very low line velocities and (2) presence of the carbon line (see also Yamanaka et al. 2009).

The velocity of the Si II $\lambda 6355$ line is $v=7,200 \mathrm{~km} \mathrm{~s}^{-1}$, lower than that of SN 2003du at a similar epoch $(10,200$ $\mathrm{km} \mathrm{s}^{-1}$ at +6.2 days $)$ and SN 2006gz $\left(11,200 \mathrm{~km} \mathrm{~s}^{-1}\right.$ at +6 days). The Si line velocity of SN 2009dc is close to that of SN $2003 \mathrm{fg}\left(8000 \mathrm{~km} \mathrm{~s}^{-1}\right.$ at +2 days, Howell et al. 2006).

The C II $\lambda 6578$ line is clearly present in the spectrum of SN $2009 \mathrm{dc}$ at +5.6 days. The velocity is $v=6,700$ $\mathrm{km} \mathrm{s}^{-1}$, which is also very low for a Type Ia SN. The C lines are rarely seen in Type Ia SNe (Marion et al. 2006; Tanaka et al. 2008b). Even if they are seen, the detection is limited almost exclusively to the very early phases: the earliest detection was in SN $1990 \mathrm{~N}$ at -15 days (Mazzali 2001), and the detection is usually at $<-10$ days for other SNe (Thomas et al. 2007). In fact, the C II line was also visible in SN 2006gz, but disappeared at -10 days. In SN 2003fg, the C II line was marginally present at +2 days.

Figure 2 shows the total flux spectrum of SN 2009dc at $t=+89.6$ days compared with SN 2003du at $t=84.0$ days (Stanishev et al. 2007). This epoch is a transition phase when the SN spectrum evolves from a photospheric spectrum to a nebular spectrum. In the spectra of both SNe, continuum emission is nearly absent, and emission features start to dominate the spectrum. Line features of SN 2009dc are narrower than those of SN 2003du even 

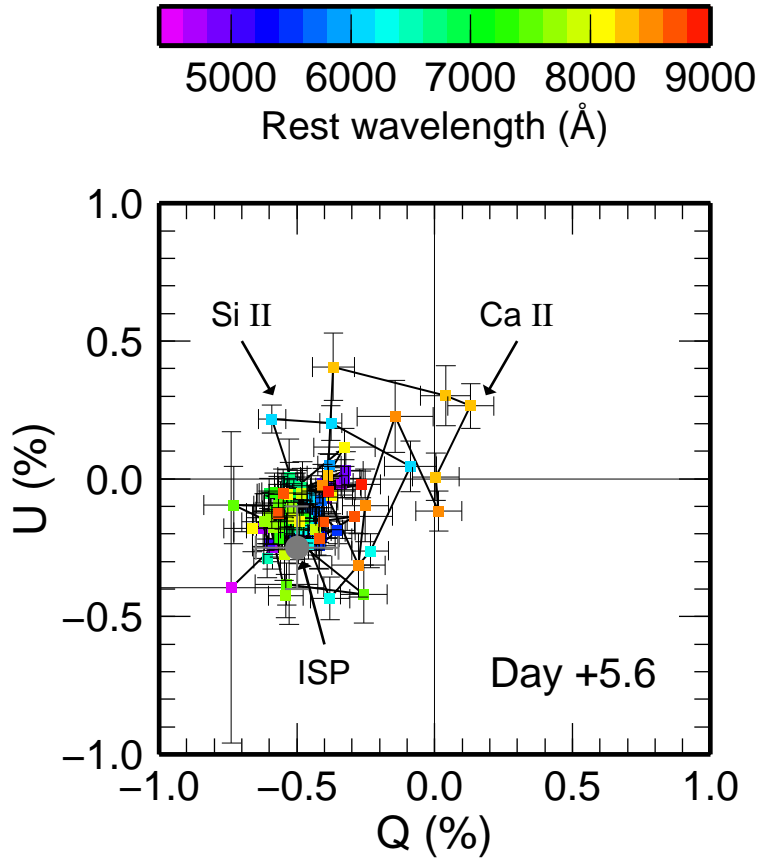

FIG. 3.- Polarization data at $t=+5.6$ days in the $Q-U$ plane. Different colors show the wavelength according to the color scale bar. ISP at $5500 \AA$ is marked with the gray point. The data are binned into $40 \AA$.

at this epoch.

\subsection{Spectropolarimetric Properties}

Observed Stokes $Q$ and $U$ at $t=+5.6$ days are shown in the middle panels of Figure 1. Polarization data are binned into $20 \AA$. It clearly shows a variation in the polarization degree at the $\mathrm{Si}$ II $\lambda 6355$ and the $\mathrm{Ca}$ II IR triplet lines. The change in the degree of polarization at these lines is $\sim 0.5 \%$ (see Section 4.2 for more details), which is broadly consistent with the past measurements for other Type Ia SNe (Wang et al. 1997, 2003a, 2007: Leonard et al. 2005; Chornock \& Filippenko 2008). A smaller fluctuation is also seen at the $\mathrm{O}$ I $\lambda 7774$.

Except for these lines, the degree of polarization is $Q \sim-0.5 \%$ and $U \sim-0.2 \%$. No significant polarization variation is detected at the $\mathrm{C}$ II line and in the wavelength region of $<6000 \AA$, where the $\mathrm{S}$ II lines and many Fe II-Fe III lines are present.

The polarization data at $t=+5.6$ days are plotted in the $Q-U$ plane in Figure 3. This also shows that the majority of the data is confined around $Q \sim-0.5 \%$ and $U \sim-0.2 \%$. And, the polarization around the Si II and $\mathrm{Ca}$ II lines deviates from this point, making a loop in the $Q-U$ plane. This is also inferred from large variation in $\theta$ at these lines (Figure 1 , see Section 4.2 for more details). According to the classification of spectropolarimetry types by Wang \& Wheeler (2008), SN 2009dc shows type N1 for the continuum (no significant dominant axis) and type L for the Si II and Ca II lines (large polarization change at the line).

Stokes $Q$ and $U$ at the Ca II IR triplet seem to have substructures, i.e., the main peak around $8300 \AA$ and a small peak at $8450 \AA$. To identify these peaks, the position of three lines $(\lambda 8498,8542,8662)$ are marked with the Doppler shifts of $v=7,200 \mathrm{~km} \mathrm{~s}^{-1}$ (photospheric component) and $v=12,000 \mathrm{~km} \mathrm{~s}^{-1}$ (high velocity component) in Figure 1]. It is found that the small peak at $8450 \AA$ originates from the photospheric component of the reddest $\mathrm{Ca}$ II $\lambda 8662$, while the main peak is a combination of the photospheric component of the other two lines and the high velocity component of the three lines. Similar substructures were also seen in the polarization spectrum of SN 2004S at +9 days (Chornock \& Filippenko 2008), but polarization of the high velocity component is smaller than the photospheric component in SN 2004S.

We also mark these two velocity components for the Si II line. Stokes $U$ has a strong peak at a similarly high velocity $\left(v=12,000 \mathrm{~km} \mathrm{~s}^{-1}\right)$ and polarization variation is seen up to $v \sim 15,000 \mathrm{~km} \mathrm{~s}^{-1}$. Note that the total flux has the absorption minimum at $v=7,200 \mathrm{~km} \mathrm{~s}^{-1}$ and does not show a strong blue wing.

Figure2 shows the polarization spectrum at $t=+89.5$ days. Given the low S/N ratio, the data are binned into $100 \AA$. The overall polarization level is similar to that at $t=+5.6$ days $(Q \sim-0.5 \%$ and $U \sim-0.2 \%)$. No significant variation (with $\gtrsim 0.5 \%$ level) of polarization is detected at the lines. Also, the polarization angle $\theta$ is nearly constant. Spectropolarimetry type at this epoch is type N1.

\subsection{Interstellar Polarization}

The spectropolarimetric data suffer from interstellar polarization (ISP) both in our Galaxy and the host galaxy. To discuss intrinsic properties of the SN polarization, ISP must be corrected. In this section, we give an estimate of ISP.

There are several possible methods to estimate ISP. When only single-epoch data are available, and if there is a dominant axis (a straight line in the $Q-U$ plane) in the polarization data, we may assume global axisymmetry of the SN ejecta and take an ISP at either end of the line in the $Q-U$ plane (Howell et al. 2001; Wang et al. 2001, 2003b; Tanaka et al. 2008a). Another method is assuming complete depolarization at strong emission lines (e.g., Kawabata et al. 2002; Leonard et al. 2005; Wang et al. 2006; Maund et al. 2007). This method is justified because the emission part of $\mathrm{P}$ Cygni profile consists largely of line-scattered, depolarized photons. However, it must be carefully done because depolarization is not necessarily complete (Tanaka et al. 2009).

To avoid the uncertainty caused by these methods, we use spectropolarimetric data taken at $t=89.5$ days. As shown in Figure 2, the spectrum at this epoch does not have clear continuum light, and electron scattering is not important. In addition, the emission features, which are intrinsically unpolarized, dominate the spectrum. Thus, the light from SN at this epoch is assumed to be intrinsically unpolarized, and the observed polarization can be considered as ISP origin.

For the wavelength dependence of ISP, we assume the following relation, which is valid for the Milky way like dust (Serkowski et al. 1975):

$$
P(\lambda)=P_{\max } \exp \left[-K \ln ^{2}\left(\lambda_{\max } / \lambda\right)\right] .
$$

Here $\lambda_{\max }$ is the wavelength at the peak of the ISP, $P_{\max }$ is the degree of the ISP at $\lambda_{\max }$, and $K$ is given as $K=$ 


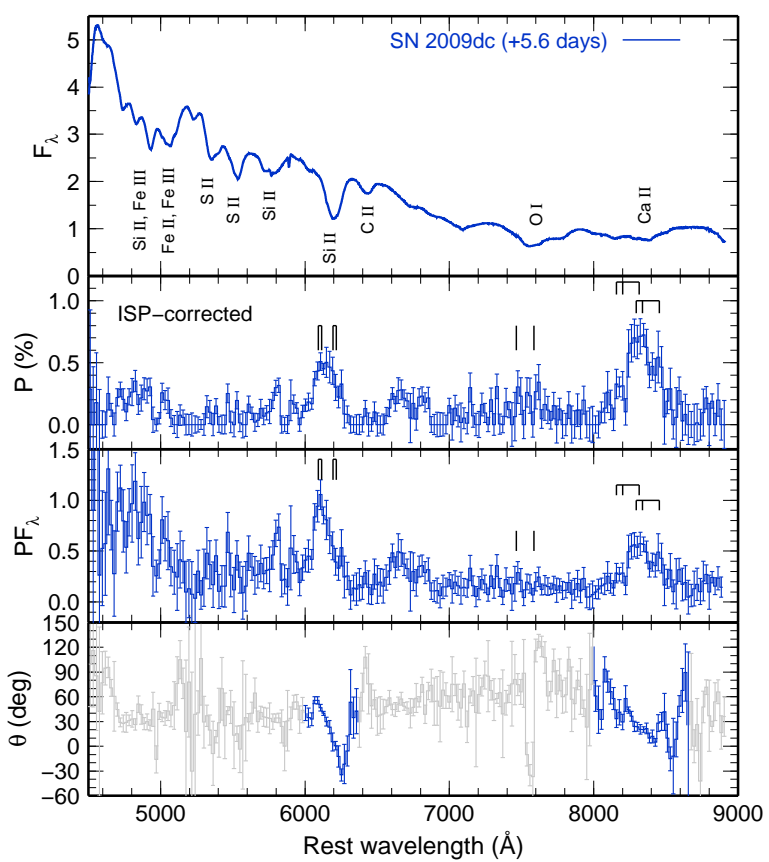

FIG. 4.- Total flux $\left(F_{\lambda}\right.$ in $\left.10^{-15} \mathrm{erg} \mathrm{s}^{-1} \mathrm{~cm}^{-2} \AA^{-1}\right)$, ISPcorrected, bias-corrected polarization $P$, polarized flux $\left(P F_{\lambda}\right.$ in $10^{-17} \mathrm{erg} \mathrm{s}^{-1} \mathrm{~cm}^{-2} \AA^{-1}$ ), and position angle $\theta$ of SN $2009 \mathrm{dc}$ at $t=+5.6$ days. In the plot of position angle, the data around the $\mathrm{Si}$ and $\mathrm{Ca}$ lines are highlighted because, except for these parts, position angle is erratically scattered and not meaningful due to the almost null polarization. Vertical lines at the Si II ( $\lambda 6347$, $6371), \mathrm{O}$ I $\lambda 7774$, and $\mathrm{Ca}$ II $(\lambda 8498,8542,8662)$ lines show the $7,200 \mathrm{~km} \mathrm{~s}^{-1}$ and $12,000 \mathrm{~km} \mathrm{~s}^{-1}$ positions.

$0.01+1.66 \lambda_{\max }(\mu \mathrm{m})$ Whittet et al. 1992). We assume $\lambda_{\max }=5500 \AA$ as a conventional choice.

The best simultaneous fit of the Stokes $Q$ and $U$ at $t=$ +89.5 days can be obtained $\left(\chi^{2} / d o f=1.1\right)$ when $Q_{\mathrm{ISP}}=$ $-0.50 \%$ and $U_{\text {ISP }}=-0.25 \%$ at $5500 \AA$, i.e., $P_{\text {ISP }, \max }=$ $0.56 \%, \theta_{\mathrm{ISP}}=103^{\circ}$. This ISP is shown in red dashed lines in Figures 1 and 2 and gray circle in Figure 3 . Note that the uncertainty of the ISP is $\sim 0.15 \%$, which is a typical error of the Stokes $Q$ and $U\left(\chi^{2} / d o f \lesssim 2.0\right.$ with this uncertainty range).

The degree of ISP is also constrained by the total extinction: $P / E(B-V)<9 \%$ (Serkowski et al. 1975). In the line of sight to SN $2009 \mathrm{dc}$, the extinction by our Galaxy is $E(B-V)_{\text {Gal }}=0.071$ mag (Schlegel et al. 1998). The extinction by the host galaxy is not certain. In our spectrum, narrow $\mathrm{Na}$ I D lines are detected, and their equivalent widths are $\mathrm{EW}=0.5$ and $1.0 \AA$ for our Galaxy and the host galaxy, respectively (these are slightly lower than the values reported by Marion et al. 2009). If we assume a host extinction proportional to the $\mathrm{EW}$, the host extinction is $E(B-V)$ host $=0.14 \mathrm{mag}$. Alternatively, if we use the relations by Turatto et al. (2003), we obtain $E(B-V)_{\text {host }}=0.15 \mathrm{mag}$ or $0.47 \mathrm{mag}$. Thus, the upper limit for the ISP is $P<0.63 \%$ in our Galaxy and $P<1.3 \%$ (tightest when $E(B-V)=0.14$ mag) in the host galaxy. The ISP estimated above is consistent with these upper limits.

\section{EXPLOSION GEOMETRY AND PROGENITOR} OF SN 2009dc

\subsection{Overall Asymmetry}

Figure 4 shows the ISP-corrected, bias-corrected total polarization $P$ at $t=5.6$ days. Assuming the ISP estimated with the later epoch data, the continuum polarization is found to be $<0.3 \%$ measured at the line-free region at $6500-7300 \AA$. Although there is a fluctuation in polarization around $6600 \AA$ (this is less significant in the original Stokes $Q$ and $U$, Figure 1D, there is no corresponding line there. Thus, we take this part as an upper limit of the continuum polarization.

The small degree of continuum polarization is consistent with other normal Type Ia SNe (e.g., Wang et al. 1997, 2003a, 2006; Leonard et al. 2005; Chornock \& Filippenko 2008). The small degree of continuum polarization indicates that the overall shape of the photosphere is nearly spherically symmetric. If a pure electron-scattering ellipsoid is assumed and if it is viewed near 90 degrees, the axis ratio of the photosphere deviates less than 10\% from unity (Höflich 1991; Wang et al. 1997).

\subsection{Element Distribution}

Variation of polarization degree at the lines is clearly detected at Si II $\lambda 6355$ and the Ca II IR triplet, and O I $\lambda 7774$ in the data taken at $t=+5.6$ days (Figure 1). The line polarization means that the distribution of these elements are not spherically symmetric. The degree of the total polarization is $0.5 \% \pm 0.1 \%$ for the $\mathrm{Si}$ II line and $0.7 \% \pm 0.1 \%$ for the Ca II line (ISP-corrected $P$, Figure 4). The polarization change at the $\mathrm{O}$ I line is affected by the choice of the ISP $(P \lesssim 0.3 \%$ in ISP-corrected $P$, Figure 41), but fluctuation in the original Stokes $Q$ and $U$ is $\sim 0.2 \%$ (Figure 1). The degree of the line polarization is similar to that of normally luminous Type Ia SNe with normal expansion velocities.

If we look at the ISP-corrected polarization (Figure 4), the smaller polarization change is also marginally seen at Si II-Fe III absorption at $4800 \AA$ and Si II $\lambda 5789$. But these are not significant, and their significance does depend on the choice of the ISP.

Polarization at the Si II, Ca II, and O I lines is plotted in the $Q-U$ plane in Figure 5, In these plots, the ISP is not corrected for, and the position of the ISP at $5500 \AA$ is shown by the gray circle. Colors of the points represent the Doppler velocity measured from $6355 \AA$ (Si II), 8567 $\AA$ (Ca II), and $7774 \AA$ (O I). As already shown in Section 3.2. polarization change at the $\mathrm{Si}$ and $\mathrm{Ca}$ lines is detected not only at photospheric velocity $\left(v=7,200 \mathrm{~km} \mathrm{~s}^{-1}\right)$, but also at higher velocities $\left(v \gtrsim 12,000 \mathrm{~km} \mathrm{~s}^{-1}\right)$. This is more clearly seen in the polarized flux $\left(P F_{\lambda}\right.$ in Figure 44), which has a peak at high velocity $\left(v \sim 12,000 \mathrm{~km} \mathrm{~s}^{-1}\right)$.

In the $Q-U$ plane (Figure 5), polarization at the Si II and $\mathrm{Ca}$ II lines shows a "loop". It can also be inferred by the large variation in the ISP-corrected position angle $\theta$ at the line (Figure (4). A similar structure in the $Q-U$ plane has been discovered in several Type Ia SNe, e.g., SN 2001el (Wang et al. 2003a) and SN 2004S (Chornock \& Filippenko 2008). The presence of the loop means that the polarization degree and position angle do depend on the velocity, i.e., the depth in the ejecta. Thus, it suggests that the distribution of the element is not axisymmetric. If the distribution were axisymmetric, the data would be distributed in a straight line in 

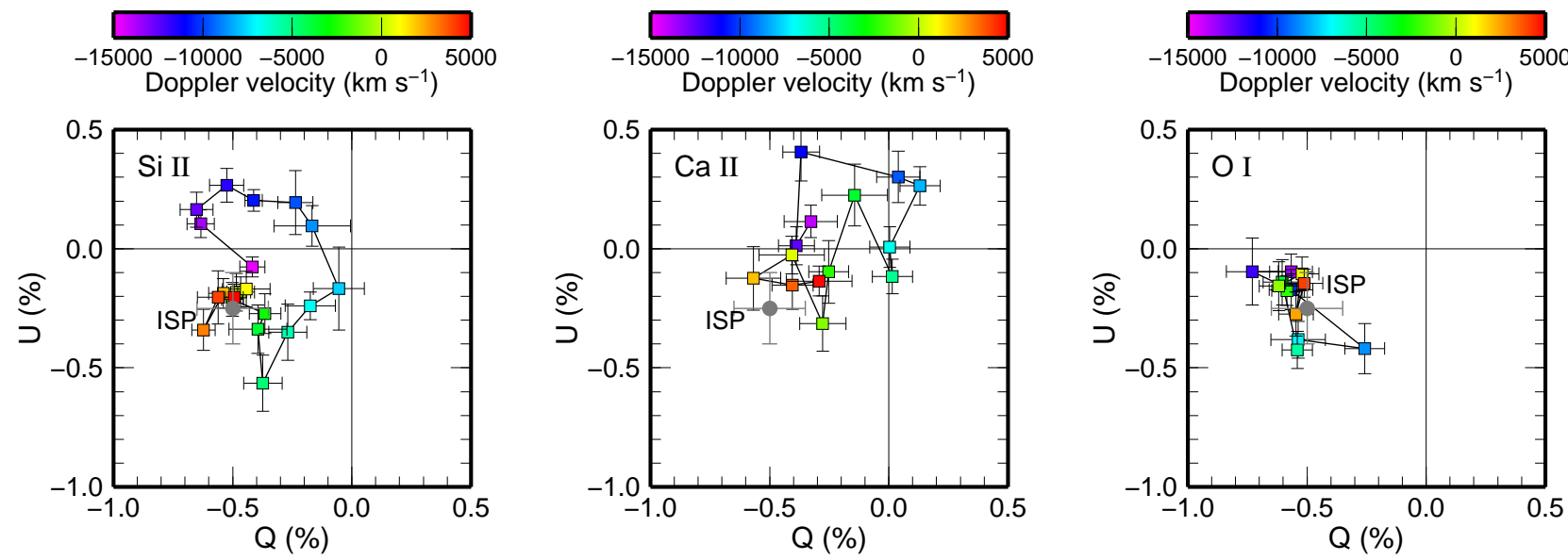

FIG. 5.- $Q-U$ diagram for the data around the Si II $\lambda 6355$, Ca II IR triplet, and O I $\lambda 7774$ at $t=+5.6$ days. Different color shows the Doppler velocity according to the color scale bar. Note that the velocity is measured from $6355 \AA$ and $8567 \AA$ for the Si II $(\lambda 6347,6371)$ and Ca II $(\lambda 8498,8542,8662)$ lines, respectively. The data are binned into $20 \AA$ for the Si II line and $40 \AA$ for the Ca II and O I lines.

the $Q-U$ plane.

Interestingly, the loop of the Si II line and the Ca II line shares a similarity: it goes counterclockwise for the larger blueshift (higher velocity, see left and center panel of Figure 5). This is in contrast to the behavior of the $\mathrm{O}$ I line, whose loop is not prominent (right panel of Figure 50. The marginal loop of the $\mathrm{O}$ I line goes to a direction different from that of the Si II and Ca II lines.

These facts suggest that the distributions of $\mathrm{Si}$ and $\mathrm{Ca}$ are correlated. In addition, the distribution of these elements is different from that of $\mathrm{O}$, which may have a complementary distribution. But all of these elements share similar velocity space. This configuration can be understood if intermediate-mass elements have a nonaxisymmetric, large-scale clumpy distribution, with some clumps sticking out to the O-rich layer (Wang et al. 2006; Wang \& Wheeler 2008).

Summarizing the spectropolarimetric properties, the explosion geometry of SN 2009dc seems to be similar to normally luminous Type Ia SNe with normal expansion velocities. The explosion is globally spherical with clumpy distribution of intermediate-mass elements.

\section{3. $P_{\mathrm{Si}}$ II $-\Delta m_{15}(B)$ Diagram}

The clumpy structure of intermediate-mass elements discussed in Section 4.2 seems to be common in Type Ia SNe (Wang et al. 2006; Wang \& Wheeler 2008; Chornock \& Filippenko 2008). In this section, we discuss the property of line polarization of SN 2009dc comparing with other Type Ia SNe.

Wang et al. (2007) presented the largest spectropolarimetric sample of Type Ia SNe. They showed a correlation between the polarization degree of the Si II line and the decline parameter of the light curve $\Delta m_{15}(B)$, the difference in the $B$-band magnitude at maximum and at 15 days after the maximum (brighter $\mathrm{SNe}$ decline more slowly, and thus, have smaller $\Delta m_{15}(B)$, e.g., Phillips 1993; Phillips et al. 1999). The data taken from Wang et al. (2007) are plotted in blue points in Figure 6. The time evolution is not corrected for. With two exceptions (SNe 2004dt and 1999by), they suggested that the brighter $\mathrm{SNe}$ (with smaller $\Delta m_{15}(B)$ ) show the smaller

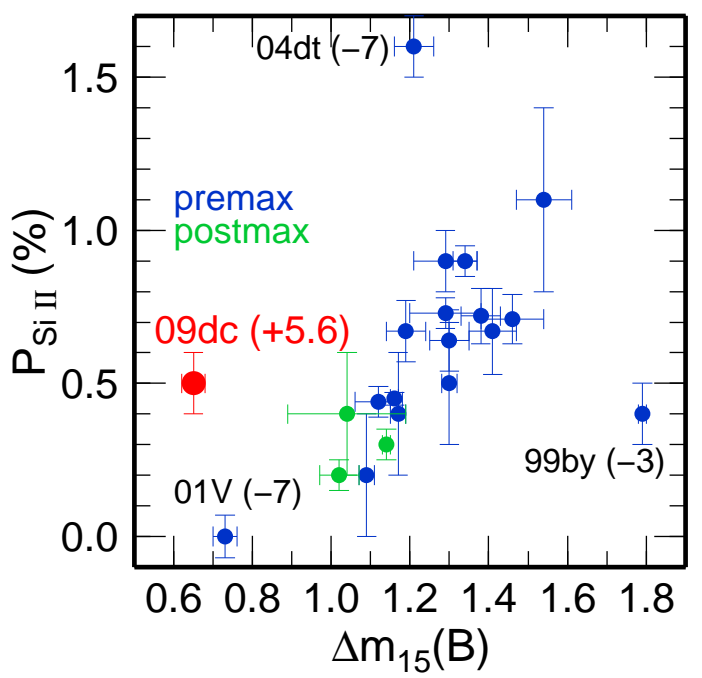

FIG. 6.- Polarization change at the Si II $\lambda 6355$ line versus the decline rate of the $B$ band light curve, $\Delta m_{15}(B)$. The red point shows SN 2009dc. The blue points show the data from Wang et al. (2007). The time evolution is not corrected for. They consist only of premaximum data (from -10 to -2 days). The green points show the postmaximum data of SNe 1997dt $(+21$ days, Leonard et al. 2005), 2003du (+18 days, Leonard et al. 2005), and 2004S (+9 days, Chornock \& Filippenko 2008).

line polarization.

Wang et al. (2007) limited their sample to the data taken at premaximum phases. From the literatures, we have added three spectropolarimetric examples at postmaximum phases (green points in Figure 6): SN $1997 \mathrm{dt}\left(+21\right.$ days, $P_{\mathrm{SiII}}=0.4 \% \pm 0.2 \%$, Leonard et al. 2005), SN 2003du (+18 days, $P_{\text {SiII }}=0.2 \% \pm 0.05 \%$, Leonard et al. 2005), and SN 2004S $\left(+4\right.$ days, $P_{\mathrm{SiII}}=$ $0.3 \% \pm 0.05 \%$, Chornock \& Filippenko 2008). Their $\Delta m_{15}(B)$ is $1.04 \pm 0.15 \mathrm{mag}$ (Jha et al. 2006), $1.02 \pm$ 0.05 mag (Anupama et al. 2005; Stanishev et al. 2007), and 1.14 $\pm 0.01 \mathrm{mag}$ (Misra et al. 2005; Krisciunas et al. 2007), respectively. We must be careful about the time 
evolution of polarization. It is thought that the degree of polarization decreases with time (Wang et al. 2003a, 2007; Patat et al. 2009), but the number of multi-epoch spectropolarimetric observations is not sufficient to clarify the common property of the time evolution.

Keeping this caveat in mind, we can still see the trend that brighter SNe have smaller line polarization. As is inferred from spectral models for Type Ia $\mathrm{SNe}$ (Mazzali et al. 2007), brigher SNe have the thinner Sirich layers, i.e., the degree of the line polarization is smaller in SNe with the thinner Si-rich layers. In addition, $\mathrm{SNe}$ with high $\mathrm{Si}$ II line velocities tend to have a high Si II line polarization (Leonard et al. 2005; Wang et al. 2006; Patat et al. 2009). In high-velocity SNe, the Si-rich layer is thought to be thick (Stehle et al. 2005; Tanaka et al. 2008b). We thus suggest that the Si line polarization can be used as an indicator of thickness of the Si-rich layers, or that enough thick Si-rich layers are required to produce the sufficient Si II line polarization.

SN 2009dc is an extremely luminous SN, synthesizing $\gtrsim 1.2 M_{\odot}$ of ${ }^{56} \mathrm{Ni}\left(\Delta m_{15}(B)=0.65 \pm 0.03 \mathrm{mag}\right.$, Yamanaka et al. 2009). Despite the large production of ${ }^{56} \mathrm{Ni}$, we have detected a moderate degree of line polarization. The degree of the Si line polarization $(0.5 \% \pm 0.1 \%$, red point in Figure 61) is higher than expected from the trend seen in other Type Ia SNe (blue and green points). This fact suggests that SN 2009dc has enough thick, clumpy Si-rich layers above $\gtrsim 1.2 M_{\odot}$ of ${ }^{56} \mathrm{Ni}$-rich layers.

It must be cautioned, however, that this discussion relies largely on the small number of spectropolarimetry of SN 1991T-like overluminous Type Ia SN, especially on SN 2001V (Wang et al. 2007, see Matheson et al. 2008 for spectroscopic behavior). To firm the conclusion, more spectropolarimetric observations of SNe with $\Delta m_{15}(B)=0.7-1.0 \mathrm{mag}$ are necessary.

\subsection{Theoretical Models}

We have shown that SN 2009dc shows the small continuum polarization and moderate line polarizations. From the relation between $\Delta m_{15}(B)$ and the Si II line polarization, it is suggested that SN 2009dc has enough thick, clumpy Si-rich layers. Combined with the low line velocities and the clear detection of the strong $\mathrm{C}$ II line (Yamanaka et al. 2009), we suggest that SN 2009dc has a super-Chandrasekhar mass.

Hillebrandt et al. (2007) suggested a possibility that the extremely luminous Type Ia SN 2003fg is an aspherical explosion of a Chandrasekhar mass white dwarf. In their scenario, the explosion is triggered at an off-center position, and nuclear burning preferentially takes place toward one direction. As a result, the distribution of ${ }^{56} \mathrm{Ni}$ is lopsided. With such a lopsided explosion, the extreme luminosity can be explained even with $0.9 M_{\odot}$ of ${ }^{56} \mathrm{Ni}$ when we observe the SN from the ${ }^{56} \mathrm{Ni}$-rich direction.

Although this explosion model is very aspherical, the expected continuum polarization may not be very large. Kasen \& Plewa (2007) presented theoretical predictions of the polarization spectra for a detonating failed deflagration model by Plewa (2007), in which the off-center ignition is followed by a surface detonation. The center of the ${ }^{56} \mathrm{Ni}$ distribution is displaced also in this model. The resultant SN can be brighter when it is viewed from the direction in which the detonation takes place. The predicted continuum polarization is small in every line of sight. This is understood as following. In the ellipsoidal geometry, non-zero polarization is expected because of the difference in the amount of light from the pole and the equator (90-degrees away), having orthogonal polarization directions. In the off-center model, on the other hand, the difference is mainly in 180-degrees away, and thus, the resultant net polarization is small.

It is also worth comparing the $\mathrm{Si}$ II line polarization of this off-center ignition model (Plewa 2007; Kasen \& Plewa 2007) with the observation $(0.5 \% \pm$ $0.1 \%)$. Although the expected polarization depends on the line of sight, Kasen \& Plewa (2007) found that the line polarization tends to be high, reflecting the aspherical distribution of intermediate mass elements. In there model, the Si II line polarization is greater than $1 \%$ in almost half of the possible viewing angles.

Next, we discuss explosion geometry of a superChandrasekhar mass white dwarf. When a superChandrasekhar mass is maintained by rapid rotation, the rotation speed is limited by the critical rotation at the surface in the equatorial direction. If the rotation is uniform, the Chandrasekhar mass is only $1.5 M_{\odot}$. If the white dwarf mass is as large as $2 M_{\odot}$, then the rotation must be differential and the white dwarf prior to the explosion has an aspherical shape (Uenishi et al. 2003; Yoon \& Langer 2005).

The explosion of super-Chandrasekhar mass white dwarf was first studied by Steinmetz et al. (1992). They explored pure detonation models and found that almost all the materials are burned into Fe-peak elements. This is incompatible with the observed properties of normal and extremely luminous Type Ia SNe.

Recently, Pfannes et al. (2010b) performed simulations of deflagration models of super-Chandrasekhar mass white dwarf. They found that the deflagration model does not result in a bright enough $\mathrm{SN}$, and is unlikely to explain the extremely luminous Type Ia SNe, as noted by Hillebrandt et al. (2007).

Interestingly, Pfannes et al. (2010a) revised the simulations of pure detonation models of superChandrasekhar mass white dwarf. They found that the entire white dwarf is not burned into Fe-peak elements, and that some intermediate mass elements are synthesized. In their models, the synthesized mass of ${ }^{56} \mathrm{Ni}$ is sufficiently large $\left(\sim 1.5 M_{\odot}\right)$ to explain the extremely luminous Type Ia SNe. It is worth testing the spectroscopic and spectropolarimetric properties of this model.

Note that a recently proposed scenario involving the collision of two white dwarfs could also realize super-Chandrasekhar mass ejecta (Rosswog et al. 2009; Raskin et al. 2009). However, this scenario predicts a very aspherical explosion, and thus, it may not be consistent with the small continuum polarization level observed in SN 2009dc.

\section{CONCLUSIONS}

We have presented the first spectropolarimetric observations of a candidate of the super-Chandrasekhar mass Type Ia SN: SN 2009dc. With the data taken at a later epoch, ISP is estimated with small uncertainty. We find that the continuum polarization of SN 2009dc is small $(<0.3 \%)$. The degree of line polarization is moderate 
$(0.5 \% \pm 0.1 \%$ at $\mathrm{Si}$ II $\lambda 6355$, and $0.7 \% \pm 0.1 \%$ at the Ca II IR triplet). These properties of polarization are similar to those of normally luminous Type Ia SNe with normal expansion velocities.

The small continuum polarization indicates that the explosion is nearly spherically symmetric. This fact suggests that a very aspherical explosion is not a likely scenario for SN 2009dc.

The polarization at the Si II and Ca II lines is detected not only at photospheric velocity $\left(7,200 \mathrm{~km} \mathrm{~s}^{-1}\right)$ but also at high velocity $\left(v \gtrsim 12,000 \mathrm{~km} \mathrm{~s}^{-1}\right)$. In addition, polarization data clearly show a loop in the $Q-U$ plane, suggesting non-axisymmetric, clumpy distribution of intermediate-mass elements.

Past spectropolarimetric observations of Type Ia SNe show the trend that brighter $\mathrm{SNe}$ have smaller line polarization (Wang et al. 2007). However, the degree of line polarization of SN 2009dc deviates from this trend: it is larger than expected from its extreme brightness. This fact suggests that there are thick enough, clumpy Si-rich layers above the thick ${ }^{56} \mathrm{Ni}$-rich layers $\left(\gtrsim 1.2 M_{\odot}\right)$, and thus, the progenitor white dwarf has a super-Chandrasekhar mass.

In summary, our spectropolarimetric observations, combined with photometric and spectroscopic observations (Yamanaka et al. 2009), suggest that SN 2009dc is an explosion of a super-Chandrasekhar mass white dwarf. The explosion geometry is globally spherically symmetric, with clumpy distribution of intermediate-mass elements.

We are grateful to the staff of the Subaru Telescope, especially Masahiko Hayashi and Hiroshi Terada for kindly allowing our observation in $\mathrm{ToO}$ time. We thank the anonymous referee for the helpful suggestions. M.T. is supported by the JSPS (Japan Society for the Promotion of Science) Research Fellowship for Young Scientists. This research has been supported in part by World Premier International Research Center Initiative, MEXT, Japan, and by the Grant-in-Aid for Scientific Research of the JSPS (18104003, 18540231, 20540226, 20840007) and MEXT (19047004, 20040004).

\section{REFERENCES}

Akerlof, C., et al. 2007, Central Bureau Electronic Telegrams, 1059 2

Anupama, G. C., Sahu, D. K., \& Jose, J. 2005, A\&A, 429, 667

Chornock, R., \& Filippenko, A. V. 2008, AJ, 136, 2227

Gerardy, C. L., et al. 2004, ApJ, 607, 391

Harutyunyan, A., Elias-Rosa, N., \& Benetti, S. 2009, Central Bureau Electronic Telegrams, 1768, 1

Hicken, M., Garnavich, P. M., Prieto, J. L., Blondin, S., DePoy, D. L., Kirshner, R. P., \& Parrent, J. 2007, ApJ, 669, L17

Hillebrandt, W., \& Niemeyer, J. C. 2000, ARA\&A, 38, 191

Hillebrandt, W., Sim, S. A., \& Röpke, F. K. 2007, A\&A, 465, L17

Höflich, P. 1991, A\&A, 246, 481

Howell, D. A., Höflich, P., Wang, L., \& Wheeler, J. C. 2001, ApJ, 556,302

Howell, D. A., et al. 2006, Nature, 443, 308

Jeffrey, D. J. 1989, ApJS, 71, 951

Jha, S., et al. 2006, AJ, 131, 527

Kasen, D., et al. 2003, ApJ, 593, 788

Kasen, D., \& Plewa, T. 2007, ApJ, 662, 459

Kashikawa, N., et al. 2002, PASJ, 54, 819

Kawabata, K. S., et al. 2002, ApJ, 580, L39

Krisciunas, K., et al. 2007, AJ, 133, 58

Landi degl'Innocenti, E. 2002, in Astrophysical Spectropolarimetry, ed. J. Trujillo-Bueno, F. Moreno-Insertis, \& F. Sánchez, 1-53

Leonard, D. C., Li, W., Filippenko, A. V., Foley, R. J., \& Chornock, R. 2005, ApJ, 632, 450

Maeda, K., \& Iwamoto, K. 2009, MNRAS, 394, 239

Maeda, K., Kawabata, K., Li, W., Tanaka, M., Mazzali, P. A., Hattori, T., Nomoto, K., \& Filippenko, A. V. 2009, ApJ, 690, 1745

Marion, G. H., Höflich, P., Wheeler, J. C., Robinson, E. L., Gerardy, C. L., \& Vacca, W. D. 2006, ApJ, 645, 1392

Marion, H., Garnavich, P., Challis, P., Calkins, M., \& Peters, W. 2009, Central Bureau Electronic Telegrams, 1776, 1

Matheson, T., et al. 2008, AJ, 135, 1598

Maund, J. R., Wheeler, J. C., Patat, F., Baade, D., Wang, L., \& Höflich, P. 2007, MNRAS, 381, 201

Mazzali, P. A. 2001, MNRAS, 321, 341

Mazzali, P. A., Röpke, F. K., Benetti, S., \& Hillebrandt, W. 2007, Science, 315, 825

McCall, M. L. 1984, MNRAS, 210, 829

Misra, K., Kamble, A. P., Bhattacharya, D., \& Sagar, R. 2005, MNRAS, 360, 662

Nomoto, K., Yamaoka, H., Shigeyama, T., Kumagai, S., \& Tsujimoto, T. 1994, in Supernovae, ed. S. A. Bludman, R. Mochkovitch, \& J. Zinn-Justin, 199-+

Oke, J. B. 1990, AJ, 99, 1621

Patat, F., Baade, D., Höflich, P., Maund, J. R., Wang, L., \& Wheeler, J. C. 2009, A\&A, 508, 229

Patat, F., \& Romaniello, M. 2006, PASP, 118, 146

Perlmutter, S., et al. 1999, ApJ, 517, 565

Pfannes, J. M. M., Niemeyer, J. C., \& Schmidt, W. 2010a, A\&A, $509, \mathrm{~A} 75+$
Pfannes, J. M. M., Niemeyer, J. C., Schmidt, W., \& Klingenberg, C. 2010b, A\&A, 509, A74+

Phillips, M. M. 1993, ApJ, 413, L105

Phillips, M. M., Lira, P., Suntzeff, N. B., Schommer, R. A., Hamuy, M., \& Maza, J. 1999, AJ, 118, 1766

Plewa, T. 2007, ApJ, 657, 942

Puckett, T., Moore, R., Newton, J., \& Orff, T. 2009, Central Bureau Electronic Telegrams, 1762, 1

Raskin, C., Timmes, F. X., Scannapieco, E., Diehl, S., \& Fryer, C. 2009, MNRAS, 399, L156

Rau, A., Kasliwal, M., \& Gal-Yam, A. 2007, Central Bureau Electronic Telegrams, 1059, 3

Riess, A. G., et al. 1998, AJ, 116, 1009

Rosswog, S., Kasen, D., Guillochon, J., \& Ramirez-Ruiz, E. 2009, ApJ, 705, L128

Scalzo, R. A., et al. 2010, ApJ, in press arXiv:1003.2217

Schlegel, D. J., Finkbeiner, D. P., \& Davis, M. 1998, ApJ, 500, 525

Schmidt, G. D., Elston, R., \& Lupie, O. L. 1992, AJ, 104, 1563

Serkowski, K., Mathewson, D. L., \& Ford, V. L. 1975, ApJ, 196, 261

Shapiro, P. R., \& Sutherland, P. G. 1982, ApJ, 263, 902

Silverman, J. M., Ganeshalingam, M., Li, W., Filippenko, A. V. Miller, A. A., \& Poznanski, D. 2010, submitted to MNRAS (arXiv:1003.2417)

Stanishev, V., et al. 2007, A\&A, 469, 645

Stehle, M., Mazzali, P. A., Benetti, S., \& Hillebrandt, W. 2005, MNRAS, 360, 1231

Steinmetz, M., Muller, E., \& Hillebrandt, W. 1992, A\&A, 254, 177

Tanaka, M., Kawabata, K. S., Maeda, K., Hattori, T., \& Nomoto, K. 2008a, ApJ, 689, 1191

Tanaka, M., et al. 2009, ApJ, 699, 1119

- . 2008b, ApJ, 677, 448

Thomas, R. C., et al. 2007, ApJ, 654, L53

Tinbergen, J. 1996, Astronomical Polarimetry (Cambridge, UK: Cambridge University Press)

Turatto, M., Benetti, S., \& Cappellaro, E. 2003, in From Twilight to Highlight: The Physics of Supernovae, ed. W. Hillebrandt \& B. Leibundgut, $200-+$

Turnshek, D. A., Bohlin, R. C., Williamson, II, R. L., Lupie, O. L., Koornneef, J., \& Morgan, D. H. 1990, AJ, 99, 1243

Uenishi, T., Nomoto, K., \& Hachisu, I. 2003, ApJ, 595, 1094

Wang, L., et al. 2003a, ApJ, 591, 1110

Wang, L., Baade, D., Höflich, P., \& Wheeler, J. C. 2003b, ApJ, 592,457

Wang, L., Baade, D., Höflich, P., Wheeler, J. C., Kawabata, K., Khokhlov, A., Nomoto, K., \& Patat, F. 2006, ÄpJ, 653, 490

Wang, L., Baade, D., \& Patat, F. 2007, Science, 315, 212

Wang, L., Howell, D. A., Höflich, P., \& Wheeler, J. C. 2001, ApJ, 550,1030

Wang, L., \& Wheeler, J. C. 2008, ARA\&A, 46, 433

Wang, L., Wheeler, J. C., \& Hoeflich, P. 1997, ApJ, 476, L27+

Whittet, D. C. B., Martin, P. G., Hough, J. H., Rouse, M. F., Bailey, J. A., \& Axon, D. J. 1992, ApJ, 386, 562

Yamanaka, M., et al. 2009, ApJ, 707, L118

Yoon, S.-C., \& Langer, N. 2005, A\&A, 435, 967 
Yuan, F., Quimby, R., Peters, C., \& Thorstensen, J. 2007, Central Bureau Electronic Telegrams, 1059, 1 